\title{
Finding Hidden Structures in Relational Databases
}

\author{
Jeffrey $\mathrm{Xu} \mathrm{Yu}$ \\ The Chinese University of Hong Kong, Hong Kong
}

\begin{abstract}
Relational database management systems have been widely used over decades. An important research issue is to find hidden structural information in large relational databases. By hidden structural information we mean the information that cannot be easily found using a traditional query language SQL. In this talk, we discuss how to find hidden structural information in a relational database by viewing a relational database as a large directed graph where nodes represent tuples and edges represent foreign key references between tuples in the database. We discuss how to find trees and communities in such a large graph for user-given keywords. We also discuss how to find frequent and additional keywords associated with the structures identified in a relational database using SQL.
\end{abstract}

